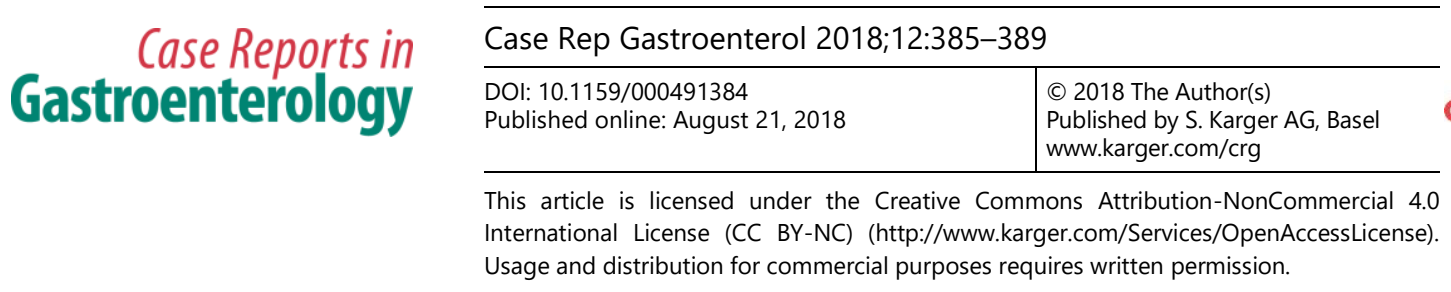

\title{
A Case of Successful Treatment of Ruptured Pancreaticoduodenal Artery Aneurysm Caused by Celiac Artery Dissection
}

\author{
Naruhiro Kimura ${ }^{a, b} \quad$ Atsunori Tsuchiya ${ }^{b} \quad$ Akihiro Nakamura $^{a}$ \\ Muneatsu Ueda $^{a}$ Seiichi Yoshikawa ${ }^{a}$ Takahiro Hoshi ${ }^{c}$ Akito Takano ${ }^{d}$ \\ Satoshi Takagi ${ }^{\mathrm{e}}$ Tsutomu Miura $^{a}$ Satoshi Yamadad ${ }^{d}$ Masahiko Yanagif \\ Tatsuo Tanig Hiroyuki Hirahara ${ }^{\text {h }}$ \\ aDivision of Gastroenterology and Hepatology, Nagaoka Red Cross Hospital, \\ Nagaoka, Japan; 'Division of Gastroenterology and Hepatology, Graduate School \\ of Medical and Dental Science, Niigata University, Niigata, Japan; 'Division of \\ Gastroenterology and Hepatology, Uonuma Institute of Community Medicine, Niigata \\ Medical and Dental Hospital, Minami-Uonuma, Japan; dDivision of Gastroenterology \\ and Hepatology, Tachikawa General Hospital, Nagaoka, Japan; 'Division of Radiology, \\ Nagaoka Red Cross Hospital, Nagaoka, Japan; fDivision of Gastroenterology and \\ Hepatology, Ojiya General Hospital, Ojiya, Japan; gDivision of Surgery, Nagaoka Red \\ Cross Hospital, Nagaoka, Japan; hDivision of Cardiovascular Surgery, Nagaoka Red Cross \\ Hospital, Nagaoka, Japan
}

\section{Keywords}

Pancreaticoduodenal artery aneurysm $\cdot$ Celiac artery dissection $\cdot \mathrm{N}$-butyl-1,2-cyanoacrylate

\section{Abstract}

A 52-year-old man was admitted due to severe epigastric lesion pain. Esophagus gastroduodenal endoscopy showed impaired duodenal dilatation, and contrast-enhanced computed tomography revealed a pancreaticoduodenal artery (PDA) aneurysm $13 \mathrm{~mm}$ in diameter below the head of the pancreas, retroperitoneal hematoma, idiopathic celiac artery (CA) dissection, and common hepatic artery disruption. Angiographic embolization with a mixture of $\mathrm{N}$-butyl1,2-cyanoacrylate and lipiodol was performed, and follow-up study showed improvement of 


\section{Case Reports in Gastroenterology}

Case Rep Gastroenterol 2018;12:385-389

DOI: $10.1159 / 000491384$

c) 2018 The Author(s). Published by S. Karger AG, Basel www.karger.com/crg

Kimura et al.: A Case of Successful Treatment of Ruptured Pancreaticoduodenal Artery Aneurysm Caused by Celiac Artery Dissection

the dilatation of the duodenum and disappearance of the aneurysm. Here we report a quite rare case of PDA aneurysm by idiopathic dissection of CA treated successfully with angiographic embolization.

\section{Introduction}

Pancreaticoduodenal artery (PDA) aneurysm is a rare condition that accounts for only $2 \%$ of all visceral aneurysms. There are two major causes for forming PDA aneurysms [1]. The first cause is inflammation, operation, and physical stimulation around the PDA, such as pancreatitis, cholecystitis, Whipple procedure, and trauma [2]. The second cause is celiac artery (CA) stenosis or occlusion such as atherosclerosis, fibromuscular hyperplasia, or median arcuate ligament (MAL) compression [3, 4]. The decrease or disruption of the blood flow of the CA causes hemodynamic changes in the blood flow of the superior mesenteric artery and especially in the PDA to supply the blood flow to the liver. This mechanism of drastic elevation of arterial pressure of the PDA is thought to cause PDA aneurysm and to result in its rupture. The incidence of PDA aneurysm rupture is reported to be 30-65\% in PDA aneurysm cases [5], and in a review of 88 PDA aneurysm patients, 53 experienced ruptures (60.2\%), which in 26 patients (49.1\%) were fatal [5]. While some of these PDA aneurysms can be detected during regular image check and be treated before rupture, some are detected incidentally or after rupture. Of the causes of CA stenosis and occlusion, idiopathic CA dissection is quite rare. Here we report a case of ruptured PDA aneurysms due to idiopathic CA dissection, with successful treatment with angiographic embolization.

\section{Case Report}

A 52-year-old man was referred to our hospital due to sudden onset of epigastric lesion pain. He had no past history of abdominal trauma, alcohol abuse, or abdominal operations, no regular medication, and no previous symptoms suggestive of pancreatitis, cholangitis, gastroduodenal ulcer, and cardiovascular disease. While his blood pressure was slightly elevated up to $154 / 100 \mathrm{~mm} \mathrm{Hg}$, other vital signs were within normal limits. Laboratory data revealed a slightly elevated white blood cell count up to 7,900 cells/ $\mu \mathrm{L}$; however, we did not detect anemia, abnormal liver panels, or abnormal pancreatic enzymes. Esophagogastroduodenoscopy showed residual food in his stomach, although his last meal had been $12 \mathrm{~h}$ before, and impaired dilatation of the third portion of the duodenum, which was a potential cause of delay of his gastric emptying (Fig. 1a). To detect the cause of impaired duodenum dilatation, contrast-enhanced computed tomography (CT) was performed and revealed an aneurysm $13 \mathrm{~mm}$ in diameter below the head of the pancreas and surrounding the retroperitoneal hematoma (Fig. 1b). The celiac trunk was narrowed by dissection from its root and dilated after dissection, in addition, the common hepatic artery was disrupted (Fig. 1c, d). The patient was diagnosed with retroperitoneal hemorrhage associated with rupture of an anterior-inferior PDA aneurysm which was caused by CA dissection. On admission, his bleeding had already stopped, and after strict blood pressure control, angiography was performed on day 5 after admission, showing blood flow from the superior mesenteric artery to the proper hepatic artery through pancreaticoduodenal arcades, and an aneurysm $15 \mathrm{~mm}$ in diameter of the anterior-inferior PDA as detected in the previous CT (Fig. 2a). Embolization was selected as treatment, and N-butyl-1,2-cyanoacrylate with lipiodol (N-butyl-1,2-cyanoacrylate and lipiodol 


\section{Case Reports in Gastroenterology}

Case Rep Gastroenterol 2018;12:385-389

DOI: $10.1159 / 000491384$

c) 2018 The Author(s). Published by S. Karger AG, Basel www.karger.com/crg

Kimura et al.: A Case of Successful Treatment of Ruptured Pancreaticoduodenal Artery Aneurysm Caused by Celiac Artery Dissection

was mixed 1:2 before injection) was injected into the anterior-inferior PDA until the hepatic artery side of the PDA was sufficiently filled (Fig. 2b, c). The patient had acute pancreatitis which improved by 2 weeks of fasting without forming pseudocyst or other complications. After treatment, esophagogastroduodenoscopy revealed improved duodenal dilatation, and contrast-enhanced CT revealed a fully embolized PDA aneurysm. Six months after admission, the patients is well without any symptoms or additional aneurysm formation under medication with nicardipine.

\section{Discussion}

Here we report a case of ruptured PDA aneurysms due to idiopathic CA dissection with successful treatment with angiographic embolization using N-butyl-1,2-cyanoacrylate with lipiodol. PDA aneurysms can be caused by chemical and physical stimulation such as pancreatitis, cholecystitis, Whipple procedure, trauma, as well as other causes, including stenosis and occlusion of the CA due to atherosclerosis and MAL compression [2]. In this case, we could not detect any of the causes mentioned above except for CA dissection; thus, we thought that CA dissection had caused the PDA aneurysm. Regarding the development mechanisms of PDA aneurysms in this case, we suspected as follows: CA dissection occurred followed by drastic increase in the pancreatic arcade that induced PDA aneurysm. In this case, the aneurysm was detected by rupture into the retroperitoneal space. In the literature, rupture of anterior-inferior PDA aneurysms can cause potentially fatal bleeding into the retroperitoneal space, abdominal cavity, gastrointestinal tract, or a combination of these. The incidence of PDA aneurysm rupture is $30-65 \%$ [5]. The size of the PDA aneurysm is not necessarily associated with the risk of rupture, and the majority of ruptured aneurysms are $<10 \mathrm{~mm}$ in diameter [6]. Regarding treatment, there are two major types of treatment: angiographic treatment and surgical treatment. Murata et al. [7] reported that ruptured aneurysms can be effectively treated by immediate embolization, which has a lower mortality rate than surgical treatment. On the other hand, Boudghène et al. [8] reported that even if the first embolization succeeded in patients with aneurysms caused by pancreatitis, bleeding recurrence was $37 \%$ and mortality $16 \%$. Mohan et al. [9] insisted that embolization is equal to surgery for these aneurysms in terms of risk, but has the advantage of more accurate anatomical arterial identification, less morbidity, and possibly lower mortality. Regarding embolization, metallic coils are used most often, while N-butyl-1,2-cyanoacrylate is rarely used because of handling difficulty and concerns regarding potentially fatal complications related to ischemic injury. However, N-butyl1,2-cyanoacrylate has benefits if the vessels are too small or too tortuous to reach the aneurysm and keep stable catheter positioning, both of which are essential for coil embolization [10]. In this case, since the artery was too tortuous, we selected angiographic embolization with the mixture of N-butyl-1,2-cyanoacrylate and lipiodol as treatment and had successful results. While acute pancreatitis and a small amount of lipiodol deposition could be detected in the liver by follow-up CT, both of these did not cause critical problems.

CA dissection is usually associated with aortic dissection. This dissection was not associated with aortic dissection, thus we considered this dissection as idiopathic CA dissection. To the best our knowledge, only 1 case with ruptured PDA aneurysm associated with CA dissection induced by MAL has been reported [11]. Although the development mechanism of idiopathic CA dissection remains unclear, Watanabe et al. [5] predicted that microtrauma from exertion or sudden abdominal hypertension can become causes of dissection. In our case, we could not diagnose the MAL by CT or angiography when the patient was admitted. If he has 
MAL syndrome, the surgery of MAL could prevent the potential risk of PDA aneurysms. Since PDA aneurysm with idiopathic CA dissection is rare, its incidence may have been underestimated due to the silent symptoms.

In conclusion, we report the successful treatment of PDA aneurysm caused by idiopathic CA dissection by angiographic embolization using N-butyl-1,2-cyanoacrylate and lipiodol without surgery. Given that PDA aneurysms have a high rupture risk and ruptured cases are often fatal, early treatment, including surgery and embolization, should be performed, whatever their size, at the time of diagnosis [12].

\section{Statement of Ethics}

The study patient's parent provided informed consent, and the study design was exempt from ethics review board approval.

\section{Disclosure Statement}

The authors declare no conflict of interest. There was no grant support.

\section{References}

1 Stanley JC, Wakefield TW, Graham LM, Whitehouse WM Jr, Zelenock GB, Lindenauer SM. Clinical importance and management of splanchnic artery aneurysms. J Vasc Surg. 1986 May;3(5):836-40.

2 Kallamadi R, Demoya MA, Kalva SP. Inferior pancreaticoduodenal artery aneurysms in association with celiac stenosis/occlusion. Semin Intervent Radiol. 2009 Sep;26(3):215-23.

3 Sutton D, Lawton G. Coeliac stenosis or occlusion with aneurysm of the collateral supply. Clin Radiol. 1973 Jan;24(1):49-53.

4 Quandalle P, Chambon JP, Marache P, Saudemont A, Maes B. Pancreaticoduodenal artery aneurysms associated with celiac axis stenosis: report of two cases and review of the literature. Ann Vasc Surg. 1990 Nov;4(6):540-5.

5 Watanabe A, Kohtake H, Furui S, Takeshita K, Ishikawa Y, Morita S. Celiac artery dissection seen with ruptured pancreaticoduodenal arcade aneurysms in two cases of celiac artery stenosis from compression by median arcuate ligament. J Vasc Surg. 2012 Oct;56(4):1114-8.

6 Flood K, Nicholson AA. Inferior pancreaticoduodenal artery aneurysms associated with occlusive lesions of the celiac axis: diagnosis, treatment options, outcomes, and review of the literature. Cardiovasc Intervent Radiol. 2013 Jun;36(3):578-87.

7 Murata S, Tajima H, Fukunaga T, Abe Y, Niggemann P, Onozawa S, et al. Management of pancreaticoduodenal artery aneurysms: results of superselective transcatheter embolization. AJR Am J Roentgenol. 2006 Sep;187(3):W290-8.

8 Boudghène F, L'Herminé C, Bigot JM. Arterial complications of pancreatitis: diagnostic and therapeutic aspects in 104 cases. J Vasc Interv Radiol. 1993 Jul-Aug;4(4):551-8.

9 Mohan IV, Stephen MS. Peripheral arterial aneurysms: open or endovascular surgery? Prog Cardiovasc Dis. 2013 Jul-Aug;56(1):36-56.

10 Won Y, Lee SL, Kim Y, Ku YM. Clinical efficacy of transcatheter embolization of visceral artery pseudoaneurysms using N-butyl cyanoacrylate (NBCA). Diagn Interv Imaging. 2015 Jun;96(6):563-9.

11 Fenoglio L, Allione A, Scalabrino E, Alberto G, Benedetti V, Pomero F, et al. Spontaneous dissection of the celiac artery: a pitfall in the diagnosis of acute abdominal pain. Presentation of two cases. Dig Dis Sci. 2004 Aug;49(7-8):1223-7.

12 de Perrot M, Berney T, Deléaval J, Bühler L, Mentha G, Morel P. Management of true aneurysms of the pancreaticoduodenal arteries. Ann Surg. 1999 Mar;229(3):416-20. 

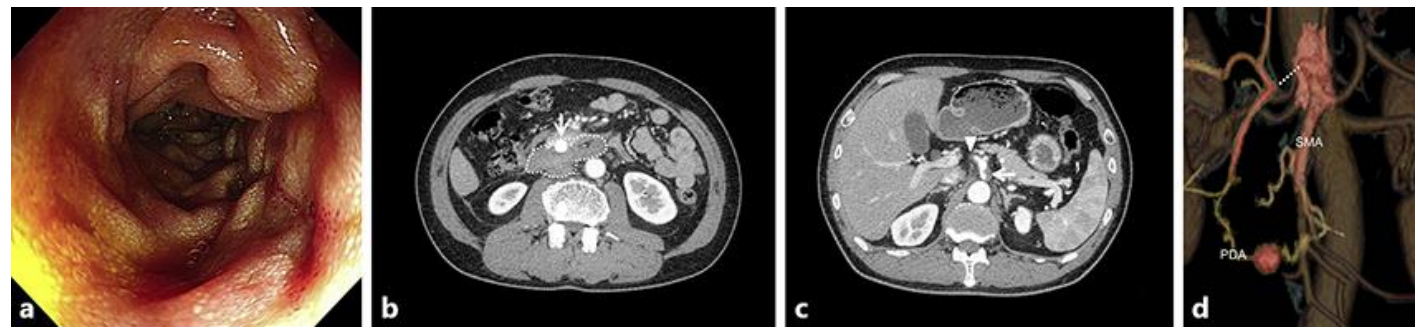

Fig. 1. Esophagogastroduodenoscopy, contrast-enhanced computed tomography, and three-dimensional image reconstruction of arteries on admission. a Esophagogastroduodenoscopy revealed that dilatation of the third portion of the duodenum was impaired. $\mathbf{b}, \mathbf{c}$ Contrast-enhanced computed tomography revealed an aneurysm which was surrounded by retroperitoneal hematoma below the head of the pancreas. $\mathbf{b}$ Arrow: aneurysm; dotted circle: hematoma. c Arrow: celiac artery dissection; arrowhead: disruption of the common hepatic artery. $\mathbf{d}$ Three-dimensional images of the artery revealed the aneurysm in the PDA. The common hepatic artery was disrupted (white dotted line). PDA, pancreaticoduodenal artery; SMA, superior mesenteric artery.
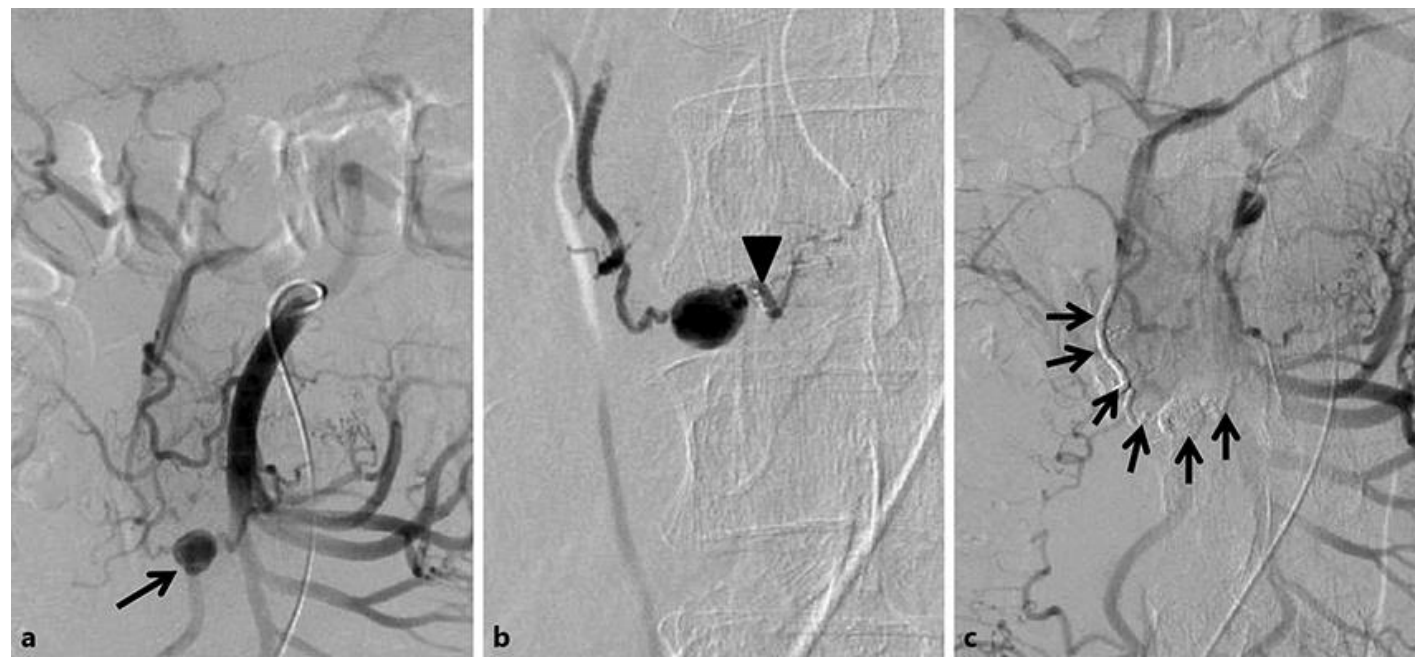

Fig. 2. Images during the angiographic treatment. a Superior mesenteric arteriogram revealed the aneurysm (arrow) in the pancreaticoduodenal artery. $\mathbf{b}$ The mixture of N-butyl-1,2-cyanoacrylate and lipiodol was injected from the site shown by the arrowhead. $\mathrm{c}$ Posttreatment superior mesenteric arteriogram revealed that the pancreaticoduodenal artery was fully filled by the mixture of N-butyl-1,2-cyanoacrylate and lipiodol (arrows). 